\title{
Long-term outcomes of coil embolization of unruptured intracranial aneurysms
}

\author{
Masaomi Koyanagi, MD, PhD, ${ }^{1}$ Akira Ishii, MD, PhD, ${ }^{2}$ Hirotoshi Imamura, MD, $\mathrm{PhD},{ }^{3}$ \\ Tetsu Satow, MD, PhD, ${ }^{4}$ Kazumichi Yoshida, MD, PhD, ${ }^{2}$ Hitoshi Hasegawa, MD, PhD, ${ }^{2}$ \\ Takayuki Kikuchi, MD, PhD, ${ }^{2}$ Yohei Takenobu, MD, PhD, ${ }^{2}$ Mitsushige Ando, MD, ${ }^{5}$ \\ Jun C. Takahashi, MD, PhD, ${ }^{4}$ Ichiro Nakahara, MD, PhD, ${ }^{5}$ Nobuyuki Sakai, MD, PhD, ${ }^{3}$ and \\ Susumu Miyamoto, MD, PhD²
}

'Department of Neurosurgery, National Hospital Organization Himeji Medical Center, Himeji; ${ }^{2}$ Department of Neurosurgery, Kyoto University Graduate School of Medicine, Kyoto; '3Department of Neurosurgery, Kobe City Medical Center General Hospital, Kobe; ${ }^{4}$ Department of Neurosurgery, National Cerebral and Cardiovascular Center, Suita; and ${ }^{5}$ Department of Neurosurgery, Kokura Memorial Hospital, Kokura, Japan

OBJECTIVE Long-term follow-up results of the treatment of unruptured intracranial aneurysms (UIAs) by means of coil embolization remain unclear. The aim of this study was to analyze the frequency of rupture, retreatment, stroke, and death in patients with coiled UIAs who were followed for up to 20 years at multiple stroke centers.

METHODS The authors retrospectively analyzed data from cases in which patients underwent coil embolization between 1995 and 2004 at 4 stroke centers. In collecting the late ( $\geq 1$ year) follow-up data, postal questionnaires were used to assess whether patients had experienced rupture or retreatment of a coiled aneurysm or any stroke or had died.

RESULTS Overall, 184 patients with 188 UIAs were included. The median follow-up period was 12 years (interquartile range 11-13 years, maximum 20 years). A total of 152 UIAs (81\%) were followed for more than 10 years. The incidence of rupture was 2 in 2122 aneurysm-years (annual rupture rate $0.09 \%$ ). Nine of the 188 patients with coiled UIAs (4.8\%) underwent additional treatment. In 5 of these 9 cases, the first retreatment was performed more than 5 years after the initial treatment. Large aneurysms were significantly more likely to require retreatment. Nine strokes occurred over the 2122 aneurysm-years. Seventeen patients died in this cohort.

CONCLUSIONS This study demonstrates a low risk of rupture of coiled UIAs with long-term follow-up periods of up to 20 years. This suggests that coiling of UIAs could prevent rupture for a long period of time. However, large aneurysms might need to be followed for a longer time.

https://thejns.org/doi/abs/10.3171/2017.6.JNS17174

KEY WORDS intracranial aneurysm; embolization; endovascular treatment; long-term follow-up; vascular disorders; interventional neurosurgery

$\mathrm{T}$ He main purpose of the treatment of unruptured intracranial aneurysms (UIAs) is to prevent rupture over the long term with low treatment risk. Although the perioperative risks of endovascular coil embolization have been repeatedly demonstrated to be less than those associated with surgical clipping, $, 1,7,10$ the long-term durability of endovascular coiling remains unclear. In the International Subarachnoid Aneurysm Trial (ISAT), the risk of rebleeding from a coiled ruptured aneurysm over 18 years was very small. ${ }^{11}$ Long-term follow-up data for prevention of rupture by endovascular coiling of UIAs, however, remain limited.

The present study aimed to evaluate the long-term effectiveness of endovascular coiling for UIAs. We analyzed the late ( $\geq 1$ year) follow-up clinical data for UIAs treated with endovascular coiling from 1995 to 2004 , followed for

ABBREVIATIONS ACA = anterior cerebral artery; ICA = internal carotid artery; IQR = interquartile range; ISAT = International Subarachnoid Aneurysm Trial; $M C A=$ middle cerebral artery; $\mathrm{mRS}=$ modified Rankin Scale; PCoA = posterior communicating artery; SAH = subarachnoid hemorrhage; UIA = unruptured intracranial aneurysm. SUBMITTED January 21, 2017. ACCEPTED June 19, 2017.

INCLUDE WHEN CITING Published online January 5, 2018; DOI: 10.3171/2017.6.JNS17174. 
up to 20 years at Kyoto University and associated stroke centers.

\section{Methods}

This study is based on criteria from the STROBE (Strengthening the Reporting of Observational Studies in Epidemiology) statement. ${ }^{17}$ The institutional review board of Kyoto University approved this observational study protocol.

\section{Data Source}

Patients with UIAs who underwent coil embolization between October 1995 and December 2004 at 4 Japanese stroke centers were retrospectively enrolled into the multicenter long-term follow-up database. This database includes patient characteristics such as age (years), sex (male or female), and modified Rankin Scale (mRS) score at the time of treatment as well as aneurysm characteristics, such as maximum size (in millimeters) and location. We also included the date of the initial procedure. This database included all of the patients who were treated in each stroke center, except 1) those who could have undergone parent artery occlusion with coils; 2) those in whom the location of the aneurysm was the cavernous portion of the internal carotid artery (ICA); and 3) those who had fusiform, traumatic, or mycotic aneurysms.

\section{Study Population}

In this study, we included all of the patients in the database except for those with 1) less than 1 year of follow-up; 2) a pretreatment $\mathrm{mRS}$ score greater than 2 ; or 3 ) incomplete data (for example, missing information on the maximum size of the aneurysm or mRS score at follow-up).

\section{Procedures}

In order to collect the follow-up data, we sent the patients a postal questionnaire that assessed their status with the self-reported mRS as well as whether they had experienced rupture of the treated aneurysms or any strokes, whether they had received any additional treatment for already-treated aneurysms during the follow-up period, and whether they were still alive. We also noted the dates of any clinical events. If the patients had already died, their families gave the date and cause of death. We collected these data via postal questionnaire from March 2016 through June 2016. If we received no response to the questionnaire, we used medical records to supplement the data.

\section{Variable Definitions}

Subarachnoid hemorrhage (SAH) was detected with CT or MRI. Stroke was defined as a change in neurological status (increase in mRS score) continuing for more than 24 hours with evidence of anatomically related lesions demonstrated with CT or MRI.

Aneurysm size (in millimeters) was measured on computer workstations using size markers or 3D rotational angiography. We classified the location of the aneurysm as ICA (including paraclinoid aneurysms [except the cavernous portion as described above], anterior choroidal ar- tery aneurysms, and terminus aneurysms), ICA-posterior communicating artery (PCoA), anterior cerebral artery (ACA; including aneurysms of the anterior communicating artery and pericallosal artery), middle cerebral artery (MCA), or posterior circulation (including aneurysms of the vertebral artery, basilar artery, cerebellar arteries, and posterior cerebral artery).

\section{Study End Points}

The primary end point was rupture of treated aneurysms throughout the late ( $\geq 1$ year) follow-up period. Secondary end points included the incidence of additional treatment for aneurysm repair procedures, any stroke that increased the mRS score for more than 24 hours, or death from any cause throughout the late follow-up period.

\section{Statistical Analysis}

Survival analyses were performed for "time to subarachnoid bleeding from the targeted aneurysm" and for "time to retreatment of the targeted aneurysm," which were measured from 1 year after the initial coil embolization. For survival analyses of retreatment, log-rank testing and multivariate Cox proportional hazards models were added. The incidence of rupture, retreatment, or stroke was assessed per aneurysm rather than per patient. The incidence of death was assessed per patient. The annual incidence of SAH was calculated by determining the number of first events divided by the number of aneurysmyears over the entire follow-up period. The annual incidence of death was calculated by determining the number of events divided by the number of patient-years over the entire follow-up period. We used a nominal $\mathrm{p}$ value of 0.05 as a threshold for statistical significance. The Mann-Whitney U-test was used for continuous variables and Fisher's exact test was used for categorical variables. All statistical analyses were performed using $\mathrm{R}$ software, version 3.2.4 (R Foundation for Statistical Computing).

\section{Results \\ Study Population}

A total of 246 patients with 252 UIAs underwent endovascular coil embolization at the 4 stroke centers between October 1995 and December 2004. Forty patients with 40 UIAs were treated in center A, 107 patients with 108 UIAs in center B, 71 patients with 75 UIAs in center $\mathrm{C}$, and 28 patients with 29 UIAs in center D, respectively. Fifty-seven patients with 58 aneurysms were excluded due to an insufficient duration of follow-up ( $<1$ year). Included in this group of 57 patients were 5 patients who died before the 1-year follow-up point; 2 of these 5 patients died due to intraoperative SAH. Data on maximum aneurysm size or mRS scores were missing for 4 additional aneurysms, which were therefore excluded from the analysis; only 3 of the 4 involved patients were excluded, however, because the fourth patient also had another aneurysm for which complete data were available. Two patients (with 2 aneurysms) were excluded because they had mRS scores greater than 2. After exclusion, 184 patients with 188 UIAs were analyzed (Fig. 1).

The median duration of follow-up was 12 years (inter- 


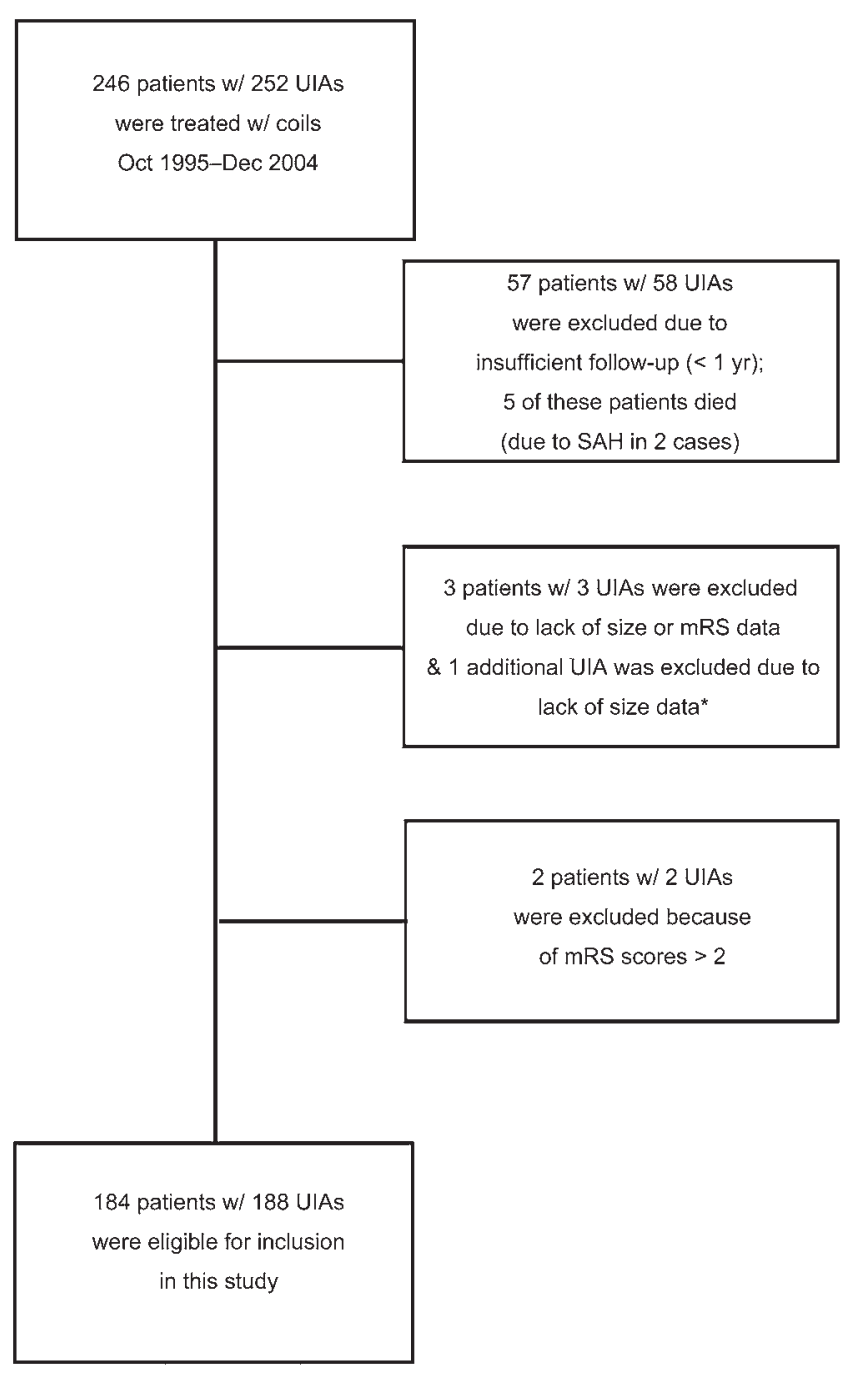

FIG. 1. Study flowchart. *One UIA that was excluded was present in a patient who also had another UIA with complete data, and who was therefore not excluded from the total number of patients.

quartile range [IQR] 11-13 years). Of the 188 UIAs, 152 $(81 \%)$ could be followed for more than 10 years. The total follow-up period was 2122 aneurysm-years. The maximum follow-up period for a single patient or aneurysm was 20 years. The baseline characteristics of the study population are shown in Table 1 .

\section{Primary End Point}

Subarachnoid hemorrhage during the late ( $\geq 1$ year) follow-up period occurred from 2 targeted aneurysms over the 2122 aneurysm-years. The annual probability of rupture of a targeted coiled aneurysm during the late followup period was $0.09 \%$. Those 2 aneurysms ruptured at day 635 and day 1655 (Fig. 2). One aneurysm was a large basilar bifurcation aneurysm that had a maximum diameter of $14 \mathrm{~mm}$. The other was a giant basilar bifurcation aneurysm that had a maximum diameter of $33 \mathrm{~mm}$. This giant aneurysm was retreated twice with coil embolization, at day 819 and again at day 1187 . There was no SAH due to
TABLE 1. Baseline characteristics of 184 patients and 188 aneurysms

\begin{tabular}{|c|c|c|}
\hline Characteristic & $\begin{array}{l}\text { Patients } \\
(n=184)\end{array}$ & $\begin{array}{c}\text { Aneurysms } \\
(n=188)\end{array}$ \\
\hline \multicolumn{3}{|l|}{ Age, yrs } \\
\hline Median (IQR) & $59(53-68)$ & $60(53-68)$ \\
\hline Mean (SD) & $59(11)$ & $59(11)$ \\
\hline \multicolumn{3}{|l|}{ Sex, n (\%) } \\
\hline Female & $146(79)$ & $150(80)$ \\
\hline Male & $38(21)$ & $38(20)$ \\
\hline \multicolumn{3}{|c|}{ mRS score before embolization, $n(\%)$} \\
\hline 0 & $170(92)$ & $173(92)$ \\
\hline 1 & $8(4)$ & $9(5)$ \\
\hline 2 & $6(3)$ & $6(3)$ \\
\hline \multicolumn{3}{|c|}{ Largest dimension of aneurysm, $\mathrm{mm}$} \\
\hline Median (IQR) & & $6(5-8)$ \\
\hline Mean (SD) & & $7.3(4.2)$ \\
\hline \multicolumn{3}{|l|}{ Distribution, $\mathrm{n}(\%)$} \\
\hline$<5 \mathrm{~mm}$ & & $40(21)$ \\
\hline $5-9 \mathrm{~mm}$ & & $112(60)$ \\
\hline$\geq 10 \mathrm{~mm}$ & & $36(19)$ \\
\hline \multicolumn{3}{|l|}{ Aneurysm location, $n(\%)$} \\
\hline ICA, except PCoA & & $98(52)$ \\
\hline Posterior circulation & & $64(34)$ \\
\hline ICA-PCoA & & $15(8)$ \\
\hline ACA & & $8(4)$ \\
\hline MCA & & $3(2)$ \\
\hline
\end{tabular}

rupture of another aneurysm. There was no SAH between the time of the procedure and 1 year later.

\section{Secondary End Point}

Overall, 9 (4.8\%) of the 188 patients with coiled UIAs underwent additional treatment in 2122 aneurysm-years. The timing of all first retreatments is shown in Fig. 3A. There were no retreatment procedures during the first 12 months after any initial treatment. Five of the 9 retreated aneurysms were first retreated at more than 5 years after initial treatment. The longest duration from initial treatment to the first retreatment procedure was 4674 days. In 3 of the 9 retreated aneurysms, additional coiling was performed twice. One aneurysm that recurred after the additional coiling was ultimately treated with clipping. In total, 12 additional coiling procedures and 1 clipping procedure were performed in this cohort. There were no neurological complications as a result of these additional treatments. Survival analysis demonstrated that posterior circulation aneurysms $(\log$-rank $\mathrm{p}=0.03)$ and large aneurysms $(\geq 10$ $\mathrm{mm}$; $\log$-rank $\mathrm{p}=0.02$ ) significantly increased the risk for retreatment of coiled UIAs (Fig. 3B). After multivariate Cox proportional hazards analyses, large aneurysm size $(\geq$ $10 \mathrm{~mm}$ ) remained a significant risk factor for retreatment (HR 4.0, 95\% CI 1.1-15, p = 0.04). In subgroup analyses, there was no significant difference in patient age between 


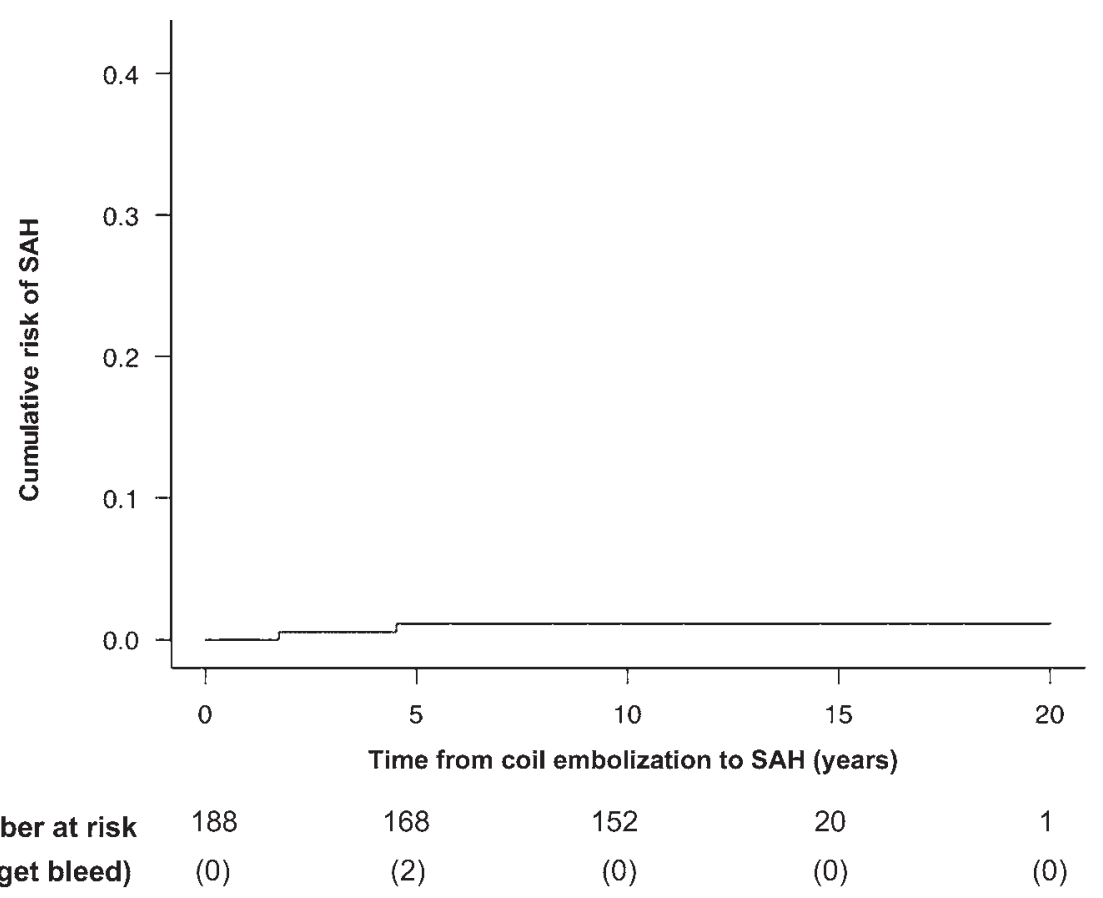

FIG. 2. Kaplan-Meier plot of cumulative risk of rupture from target aneurysm.

the groups of aneurysms that were retreated more than 5 years or less than 5 years after initial treatment (MannWhitney U-test, $\mathrm{p}=0.41)$, large aneurysms (OR 0.28, 95\% CI $0.003-5.9, \mathrm{p}=0.55$ ), or posterior circulation aneurysms (OR 1.4, 95\% CI 0.08-31, $\mathrm{p}=1.0$ ).

Strokes that decreased the mRS score occurred in 9 patients with 9 aneurysms over 2122 aneurysm-years. Among the 9 strokes during the late follow-up period, 6 were ischemic, 2 were SAHs as described above, and 1 was an intracerebral hemorrhage.

During the 20-year follow-up period, 17 (9.2\%) of 184 patients died over 2074 patient-years. The annual probability of death was $0.82 \%$. Two (12\%) of the 17 deaths were related to SAH. Five of the deaths were due to cardiovascular disease and an additional 5 were due to cancer.

\section{Discussion}

In this observational study, we provided long-term multicenter follow-up data on the clinical outcomes of coil embolization for UIAs. The risk of rupture from a coiled aneurysm during the late ( $\geq 1$ year) follow-up period was small (annual rate 0.09\%). The rate of requiring retreatment of a coiled aneurysm was relatively low (4.8\%). However, more than half of the retreatments were performed more than 5 years after the initial treatment.

Long-term follow-up data on the risk of SAH after coil embolization for UIAs are considered to be important but limited. ${ }^{2}$ The mean follow-up period in Naggara and colleagues' systematic review of coiled UIAs ranged from 0.4 to 3.2 years and was limited to the first 6 months for three-fourths of reported cases. ${ }^{13}$ An observational study of a California database of 944 patients with UIA treated with endovascular coiling showed long-term results over a median follow-up period of 7 years. ${ }^{5}$ However, because that study provided only the incidence of nontraumatic intracranial hemorrhage, the incidence of aneurysmal SAH after coil embolization was not available. In our study, the median follow-up period was 12 years, and the longest follow-up period was 20 years. Our long-term data could provide one possible answer to the question of whether the preventive effects of endovascular coiling for UIA rupture could last for an extended period of time.

In the ISAT, which included only SAH cases, longterm follow-up data ( $>10$ years) showed that rebleeding occurred from coiled aneurysms in 13 subjects over 8351 patient-years (annual rate $0.15 \%$ ). ${ }^{11}$ These data provided the incidence of rerupture 1 year after the initial treatment. That incidence of posttreatment rupture (i.e., rerupture) seems relatively high compared with the incidence of posttreatment rupture (i.e., initial rupture) in our study. A possible explanation may lie in the pretreatment status of the aneurysms in the 2 studies (ruptured vs unruptured). The possibility of recanalization appears greater in ruptured aneurysms than in UIAs, ${ }^{14}$ and this might explain the greater risk of posttreatment rupture in the ISAT.

Our results show that more than half of aneurysm retreatments were performed more than 5 years after the initial treatment. Ferns et al. ${ }^{4}$ showed that aneurysm reopening usually occurs early after coil embolization and concluded that "prolonged imaging follow-up" may not be beneficial with respect to detection of reopened aneurysms that require additional treatment, which conflicts with our findings. In their study, Ferns and colleagues assessed the incidence of late ( $>4.5$ years) aneurysm reopening after coil embolization in a series of 400 patients with 440 aneurysms using 3-T MR angiography. Delayed aneurysm reopening occurred in 11 patients $(2.8 \%)$, with only 3 lesions $(0.7 \%)$ requiring further treatment. One pos- 

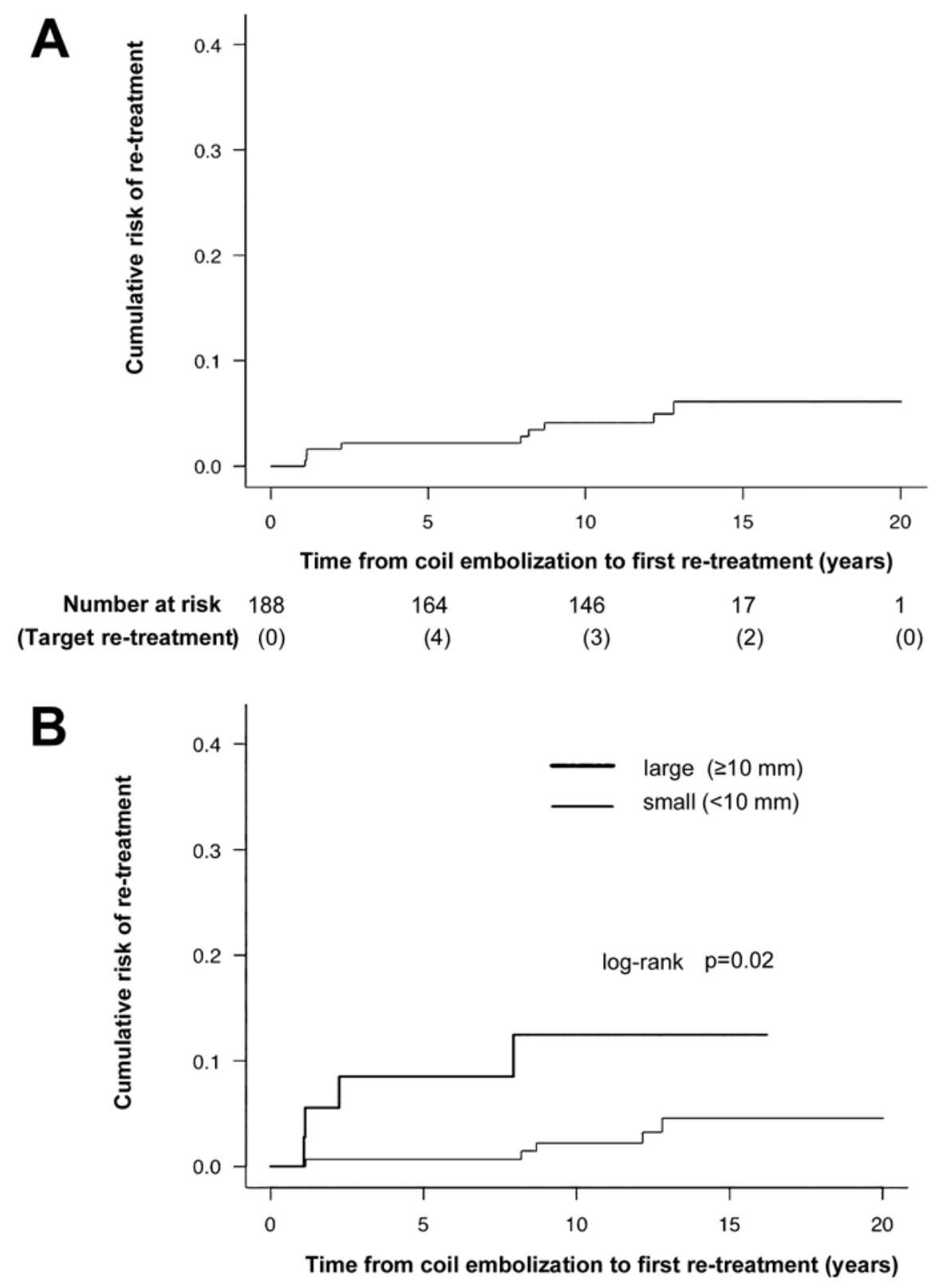

Number at risk

$\begin{array}{llllll}\text { large }(\geq 10 \mathrm{~mm}) & 36 & 27 & 21 & 2 & 0 \\ \text { small }(<10 \mathrm{~mm}) & 152 & 137 & 125 & 15 & 1\end{array}$

FIG. 3. A: Kaplan-Meier plot of overall cumulative risk of retreatment of target aneurysm. B: Kaplan-Meier plot of cumulative risk of retreatment of aneurysm according to size.

sible explanation for the difference in the findings in the 2 studies is the lengths of the follow-up periods. The median duration in the study by Ferns et al. was 5 years, whereas the median duration in our study was 12 years. In another recent study, Chalouhi et al. ${ }^{2}$ found that the sensitivity of short-term follow-up studies was only $40 \%$ for detecting delayed aneurysm recurrence. In that study, the authors evaluated short- and long-term ( $>3$ years) follow-up digital subtraction angiography images obtained in 209 patients who had undergone coil embolization of ruptured or unruptured aneurysms. Short-term follow-up images showed minor recurrence in $25 \%$ of cases, with none requiring retreatment, whereas long-term follow-up images showed recurrence in over $40 \%$ of patients who had adequate aneurysm occlusion, with $26 \%$ requiring retreatment. This may support our findings about the importance of long-term follow-up.

Another finding about retreatment in our study was that large aneurysms $(\geq 10 \mathrm{~mm})$ significantly increase the risk for retreatment. Large size is a well-known risk factor for reopening of coiled aneurysms. ${ }^{3,16}$ Although without firm guidelines it is difficult or nearly impossible to objectively 
determine whether a reopened aneurysm should be retreated, ${ }^{8}$ according to our data and a previous report ${ }^{5}$ about retreatment, long-term follow-up could be recommended, especially for large coiled aneurysms.

Our study also had limitations. First, there was selection bias regarding the choice of endovascular coiling for the treatment of UIAs. Historically, the primary indication for endovascular coiling of a UIA was in patients for whom surgery was considered to be high risk. As a result, in this cohort, a higher proportion of included aneurysms were non-PCoA ICA aneurysms and posterior circulation aneurysms. Because ICA aneurysms (other than ICA-PCoA aneurysms) were shown to have a lower rate of rupture in the International Study of Unruptured Intracranial Aneurysms (ISUIA) ${ }^{18}$ and the Unruptured Cerebral Aneurysm Study of Japan (UCAS Japan), ${ }^{12}$ this might have influenced our clinical results.

Second, this study included only Japanese patients. Reportedly, Japan has a higher incidence of SAH than other countries, except for Finland. ${ }^{6}$ The UCAS Japan study showed a higher risk of rupture than that shown in the ISUIA. ${ }^{12,18}$ Although the rupture risk of coiled UIAs is considered to be different from that of untreated UIAs, this ethnic issue may have affected our results.

Third, we excluded 64 aneurysms from this study; 58 were excluded because we had less than 1 year of followup data for those lesions. There is a possibility that the excluded cases may have affected our results. However, there was no rupture or retreatment of aneurysms during these short follow-up periods.

Fourth, in this study, most aneurysms were treated with older-generation devices. We did not use neck-bridging stents that could have achieved better long-term anatomical results. ${ }^{15}$ The recent development of new endovascular treatment might improve long-term outcomes compared with our results. However, we should keep in mind that new technological advancements have widened treatment indications for complex UIAs that had previously been considered to be unsuitable for coiling.

The final limitation of the study is the retrospective nature of the data. Clinical follow-up was not complete in all patients. There is a possibility that some patients with SAH were missed.

\section{Conclusions}

Our observational study demonstrates a low risk for rupture of coiled UIAs with longer follow-up periods of up to 20 years. This suggests that the effectiveness of coiling UIAs for the prevention of aneurysm rupture could last for an extended period. However, more than half of the retreatments were performed more than 5 years after the initial treatment. Additionally, large aneurysm size was the most significant risk factor for retreatment. These data suggest that long-term follow-up might be needed for patients who have undergone coil embolization of large UIAs.

\section{References}

1. Brinjikji W, Rabinstein AA, Nasr DM, Lanzino G, Kallmes DF, Cloft HJ: Better outcomes with treatment by coiling relative to clipping of unruptured intracranial aneurysms in the United States, 2001-2008. AJNR Am J Neuroradiol 32:1071-1075, 2011

2. Chalouhi N, Bovenzi CD, Thakkar V, Dressler J, Jabbour P, Starke RM, et al: Long-term catheter angiography after aneurysm coil therapy: results of 209 patients and predictors of delayed recurrence and retreatment. J Neurosurg 121:11021106, 2014

3. Ferns SP, Sprengers MES, van Rooij WJ, Rinkel GJE, van Rijn JC, Bipat S, et al: Coiling of intracranial aneurysms: a systematic review on initial occlusion and reopening and retreatment rates. Stroke 40:e523-e529, 2009

4. Ferns SP, Sprengers MES, van Rooij WJ, van Zwam WH, de Kort GA, Velthuis BK, et al: Late reopening of adequately coiled intracranial aneurysms: frequency and risk factors in 400 patients with 440 aneurysms. Stroke 42:1331-1337, 2011

5. Gonda DD, Khalessi AA, McCutcheon BA, Marcus LP, Noorbakhsh A, Chen CC, et al: Long-term follow-up of unruptured intracranial aneurysms repaired in California. J Neurosurg 120:1349-1357, 2014

6. Greving JP, Wermer MJ, Brown RD Jr, Morita A, Juvela S, Yonekura M, et al: Development of the PHASES score for prediction of risk of rupture of intracranial aneurysms: a pooled analysis of six prospective cohort studies. Lancet Neurol 13:59-66, 2014

7. Hoh BL, Chi YY, Lawson MF, Mocco J, Barker FG II: Length of stay and total hospital charges of clipping versus coiling for ruptured and unruptured adult cerebral aneurysms in the Nationwide Inpatient Sample database 2002 to 2006. Stroke 41:337-342, 2010

8. Kallmes DF, Cloft HJ: Ready or not, here they come: randomized trials evaluating new endovascular aneurysm therapies. AJNR Am J Neuroradiol 28:799-803, 2007

9. McDonald JS, McDonald RJ, Fan J, Kallmes DF, Lanzino G, Cloft HJ: Comparative effectiveness of unruptured cerebral aneurysm therapies: propensity score analysis of clipping versus coiling. Stroke 44:988-994, 2013

10. Molyneux A, Kerr R, Stratton I, Sandercock P, Clarke M, Shrimpton J, et al: International Subarachnoid Aneurysm Trial (ISAT) of neurosurgical clipping versus endovascular coiling in 2143 patients with ruptured intracranial aneurysms: a randomised trial. Lancet 360:1267-1274, 2002

11. Molyneux AJ, Birks J, Clarke A, Sneade M, Kerr RS: The durability of endovascular coiling versus neurosurgical clipping of ruptured cerebral aneurysms: 18 year follow-up of the UK cohort of the International Subarachnoid Aneurysm Trial (ISAT). Lancet 385:691-697, 2015

12. Morita A, Kirino T, Hashi K, Aoki N, Fukuhara S, Hashimoto N, et al: The natural course of unruptured cerebral aneurysms in a Japanese cohort. N Engl J Med 366:2474-2482, 2012

13. Naggara ON, White PM, Guilbert F, Roy D, Weill A, Raymond J: Endovascular treatment of intracranial unruptured aneurysms: systematic review and meta-analysis of the literature on safety and efficacy. Radiology 256:887897,2010

14. Nguyen TN, Hoh BL, Amin-Hanjani S, Pryor JC, Ogilvy CS: Comparison of ruptured vs unruptured aneurysms in recanalization after coil embolization. Surg Neurol 68:19-23, 2007

15. Nishido H, Piotin M, Bartolini B, Pistocchi S, Redjem H, Blanc R: Analysis of complications and recurrences of aneurysm coiling with special emphasis on the stent-assisted technique. AJNR Am J Neuroradiol 35:339-344, 2014

16. Sluzewski M, Menovsky T, van Rooij WJ, Wijnalda D: Coiling of very large or giant cerebral aneurysms: long-term clinical and serial angiographic results. AJNR Am J Neuroradiol 24:257-262, 2003

17. von Elm E, Altman DG, Egger M, Pocock SJ, Gøtzsche PC, Vandenbroucke JP : The Strengthening the Reporting of Observational Studies in Epidemiology (STROBE) state- 
ment: guidelines for reporting observational studies. Lancet 370:1453-1457, 2007

18. Wiebers DO, Whisnant JP, Huston J III, Meissner I, Brown RD Jr, Piepgras DG, et al: Unruptured intracranial aneurysms: natural history, clinical outcome, and risks of surgical and endovascular treatment. Lancet 362:103-110, 2003

\section{Disclosures}

Dr. Ishii reports a grant from Medtronic and speaker fee from Medtronic, outside the submitted work. Dr. Nakahara reports grants from Terumo and Kaneka Medix and speaker fees from Stryker, Johnson \& Johnson, Medtronic, Terumo, and Kaneka Medix, outside the submitted work. Dr. Sakai reports a grant from Terumo and speaker fees from Stryker, Terumo, Medtronic, and Johnson \& Johnson, outside the submitted work.

\section{Author Contributions}

Conception and design: Koyanagi, Ishii, Imamura, Satow, Yoshida, Hasegawa, Sakai, Miyamoto. Acquisition of data: Imamura, Satow, Kikuchi, Ando. Analysis and interpretation of data: Koyanagi, Ishii, Imamura, Satow, Yoshida, Kikuchi, Takenobu, Sakai, Miyamoto. Drafting the article: Koyanagi. Critically revising the article: Imamura, Nakahara, Miyamoto. Reviewed submitted version of manuscript: Ishii, Imamura, Satow, Yoshida, Hasegawa, Kikuchi, Takenobu, Ando, Takahashi, Nakahara, Sakai, Miyamoto. Approved the final version of the manuscript on behalf of all authors: Koyanagi. Statistical analysis: Koyanagi. Administrative/technical/material support: Koyanagi, Takahashi, Nakahara, Sakai, Miyamoto. Study supervision: Ishii, Sakai, Miyamoto.

\section{Correspondence}

Masaomi Koyanagi: National Hospital Organization Himeji Medical Center, Himeji, Hyogo, Japan. koyanagm@gmail.com. 